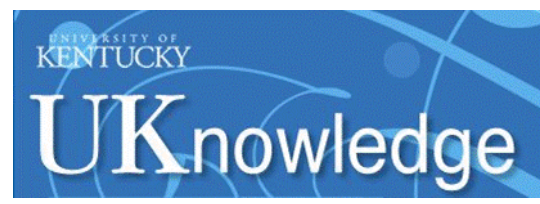

University of Kentucky

UKnowledge

$11-1-1984$

\title{
Cosmic Rays and the Emission-Line Regions of Active Galactic Nuclei
}

\author{
Gary J. Ferland \\ University of Kentucky, gary@uky.edu \\ R. F. Mushotzky \\ NASA/Goddard Space Flight Center
}

Follow this and additional works at: https://uknowledge.uky.edu/physastron_facpub

Part of the Astrophysics and Astronomy Commons, and the Physics Commons

Right click to open a feedback form in a new tab to let us know how this document benefits you.

\section{Repository Citation}

Ferland, Gary J. and Mushotzky, R. F., "Cosmic Rays and the Emission-Line Regions of Active Galactic Nuclei" (1984). Physics and Astronomy Faculty Publications. 177.

https://uknowledge.uky.edu/physastron_facpub/177

This Article is brought to you for free and open access by the Physics and Astronomy at UKnowledge. It has been accepted for inclusion in Physics and Astronomy Faculty Publications by an authorized administrator of UKnowledge. For more information, please contact UKnowledge@lsv.uky.edu. 
Cosmic Rays and the Emission-Line Regions of Active Galactic Nuclei

Digital Object Identifier (DOI)

http://dx.doi.org/10.1086/162574

Notes/Citation Information

Published in The Astrophysical Journal, v. 286, no. 1, p. 42-52.

(c) 1984. The American Astronomical Society. All rights reserved.

The copyright holder has granted permission for posting the article here.

This article is available at UKnowledge: https://uknowledge.uky.edu/physastron_facpub/177 
The Astrophysical Journal, 286:42-52, 1984 November 1

(C) 1984. The American Astronomical Society. All rights reserved. Printed in U.S.A

COSMIC RAYS AND THE EMISSION-LINE REGIONS OF ACTIVE GALACTIC NUCLEI

\author{
G. J. FERLAND \\ Department of Physics and Astronomy, University of Kentucky, Lexington \\ AND \\ R. F. MUSHOTZKY \\ Laboratory for High Energy Astrophysics, NASA/Goddard Space Flight Center \\ Received 1983 March 10; accepted 1984 May 3
}

\begin{abstract}
We discuss the effects that the synchrotron emitting relativistic electrons, which are known to exist throughout many distance scales in active nuclei, could have on the emission-line regions that characterize these objects. Detailed models of both the inner, dense, broad-line region and the outer, lower density, narrow-line region are presented, together with the first models of the optically emitting gas often found within extended radio lobes. We show that, in all cases, if the relativistic gas that produces the synchrotron radio emission is mixed with the gas in the emission-line region, then significant changes in the emission-line spectrum will result. For small cosmic-ray densities the main effect is to strengthen lines that are formed in neutral regions, where photoelectric heating rates are lowest. As the cosmic-ray density increases, so do both the temperature and the ionization, until the cooling peak near $10^{5} \mathrm{~K}$ is reached and a thermal runaway occurs.

The implications of our results for correlations between radio and optical properties of nuclei are discussed. We find that the addition of a flux of cosmic rays to a standard model of the broad-line region can effectively quench $\mathrm{Fe}$ II emission, in agreement with Grandi and Osterbrock's discovery that radio-loud objects tend to be weak $\mathrm{Fe}$ II emitters. The models do not reproduce their correlation between radio properties and Balmer decrements. Models of low-density gas in the narrow-line region show that relativistic particles can raise the temperature in the $[\mathrm{O}$ III] zone by an amount sufficient to explain some observed $\lambda 5007 / \lambda 4363$ ratios. Although the addition of a flux of cosmic rays is probably not the only way to explain these anomalies in the emission spectra of broad- and narrow-line regions, it does provide a mechanism that is both simple and natural for powering the optically emitting extended regions that lie in the extended radio lobes far from the central engine.

The effects of the synchrotron emitting electrons on filaments in the Crab Nebula are discussed in an appendix, along with a comparison between our calculations, which employ the mean escape probability formalism, and recent Hubbard and Puetter models.
\end{abstract}

Subject headings: BL Lacertae objects - galaxies: nuclei - nebulae: Crab Nebula - nebulae: general radiation mechanisms - relativity

\section{INTRODUCTION}

In general, active galactic nuclei (AGNs) such as radio and Seyfert galaxies, as well as the more luminous quasars and QSOs, are characterized by emission-line spectra that are the composite of several distinct regions (see Shields 1974, as well as the review by Davidson and Netzer (1979). Photoionization models have been quite successful in reproducing the general features of the ultraviolet, optical, and infrared spectrum (e.g., Kwan and Krolik 1981; Péquignot 1984). However, many members of the class of AGNs (the radio galaxy and quasar subclasses) are powerful synchrotron radio sources, and this emission is generally taken as an indication of the presence of particles with relativistic energies (see Pacholczyk 1970). These particles could have dramatic effects on the cold clouds in the emission-line region. In general, it is not known whether these cosmic rays actually mix with the cold gas, although it is known that both optical and radio emission occur on the same distance scales.

It is not our intention to challenge the standard assumption that photoionization is an important heating and ionization process for emission-line regions of these objects; indeed, the entire class of AGNs are known to be sources of relatively hard radiation (Mushotzky 1982), and photoionization must occur.
Rather, it is our goal to examine the effects of a modest additional heating and ionization agent in the form of the relativistic particles that are known to exist within these sources. Our motivation in this work is the discovery by Grandi and Osterbrock (1978) that the optical spectra of radio galaxies are systematically different from those of the radio-quiet Seyfert galaxies. Apparently, the radio properties of the central engine are somehow communicated to the surrounding emission-line gas. This raises the question: What effects will the cosmic rays that produce the radio emission have on the emission-line clouds?

We know of no discussion of the effects of such a cosmic-ray flux upon standard photoionization models of AGN emissionline regions. Mechanical energy input in the form of shock waves (see Dopita 1977; Daltabuit, MacAlpine, and Cox 1978) has been considered for both supernova remnants and AGNs, and a model invoking particle streams with subsequent chargeexchange neutralization (see Ptak and Stoner 1973) has been worked out in some detail. Baldwin et al. (1977) have considered some properties of a model in which a flux of cosmic rays interacts with the emission-line regions, although they were interested primarily in setting limits on the cosmic-ray flux near quasars from the absence of spallation products. 
Krolik, McKee, and Tarter (1981) discussed the effects of relativistic particles on a hot intercloud medium, and set limits to the amount of extra heating a cold cloud could tolerate. Balbus and McKee (1982) have discussed the stability of spherical clouds to disruption by energetic particles, while Aldrovandi and Péquignot (1973) have discussed the general case of cosmic-ray heating. Cosmic-ray heating is also thought to be important in heating the hot intergalactic gas found in many clusters (Sofia 1973; Lea and Holman 1978; Tucker and Rosner 1983).

Section II presents some details of the calculations we have performed. We use a standard photoionization code that has been modified to include both heating and ionization through the interaction of the relatively cold gas with energetic particles. In § III we discuss some detailed models of the various emission-line regions of active nuclei, and show how the cosmic rays will affect the photoionized gas if the two mix. Section IV presents a discussion of the implications of our results.

A discussion of the effects of cosmic rays on a filament in the Crab Nebula, and a comparison between our predictions and those of Hubbard and Puetter (1984) for a quasar emission-line region, are found in appendices.

\section{MODEL CALCULATIONS}

Our goal is to determine the effects of a flux of high-energy particles, such as that responsible for the observed radio emission, on three specific models. We begin by describing the generally accepted model for the production of both radio continuum and optical emission-line production in AGNs, and then give some details of the method used to model these nebulae.

\section{a) The Emission Regions}

The optical spectra of most AGNs show evidence of at least two, and perhaps three, emission-line regions. The evidence for this breakdown is summarized by Shields (1974), as well as by Osterbrock (1984). Both dynamical and energy-balance arguments place the dense, so-called broad-line region (BLR) at a distance of roughly a few light months from the central object, while a lower density, narrow-line region (NLR) is considerably more distant $\left(r \sim 10^{2}-10^{3} \mathrm{pc}\right)$. The fact that the NLR [O III ] lines indicate a density inconsistent with the presence of the familiar [O II] and [S II] lines (see Osterbrock 1981) suggests that the NLR actually has at least two components (cf. Netzer; 1982; Péquignot 1984). In addition to these prominent emission regions, careful scrutiny will often reveal faint, very low density emission regions within the extended radio lobes $\left(r \sim 10^{4} \mathrm{pc}\right.$; cf. Saslaw, Tyson, and Crane 1978; van Breugel et al. 1984).

Radio emission also occurs on all these distance scales (cf. Osterbrock 1984; Wilson 1983; Ulvestad, Wilson, and Sramek 1981). The density of the relativistic particles producing the radio emission can be estimated from the minimum-energy argument (Burbidge, Jones, and O'Dell 1974), and these range from $N^{*} \sim 10^{-2} \mathrm{~cm}^{-3}$ up to $N^{*} \sim 10^{8} \mathrm{~cm}^{-3}$ for the compact component near the BLR, to $N^{*} \sim 10^{-6} \mathrm{~cm}^{-3}$ for extended radio lobes. Although it is known that the radio emission tends to favor an axial symmetry (which may or may not be aligned with the symmetry axis of the host galaxy; see Tohline and Osterbrock 1982), it is not known how this axis is positioned relative to the emission-line regions. This uncertainty is due in large part to a nearly complete ignorance of the geometry and kinematic state of the cold gas in the inner regions.

\section{b) Photoionization Calculations}

Photoionization calculations are performed with a modified version of the program described by Ferland and Mushotzky (1982). Briefly, a slab of gas is divided into a large number of zones (typically $\sim 100-200$ ), and the equations of statistical and thermal equilibrium are solved for each zone. The ionizing continuum is modified by both extinction due to the photoelectric, bremsstrahlung, electron scattering, and absorption-line opacities, and reemission by the gas (treated in the outwardonly formalism in the first iteration, and by the so-called lambda-iteration method in subsequent iterations [see Williams 1967; Mihalas 1978]). Williams (1967), Davidson (1972), MacAlpine (1972), and especially Davidson and Netzer (1979) offer further elaboration.

The present calculations include the "state of the art" techniques and processes described by Kwan and Krolik (1981), Henry and MacAlpine (1982), Halpern and Grindlay (1980), and Kallman and McCray (1982). Line ransfer is treated by the escape probability approximation as developed by Kwan and Krolik (1981). Hydrogen is treated with seven levels ( $2 s$ and $2 p$ are treated independently) and continuum, and escape probabilities are given by

$$
\left.\begin{array}{rlrl}
\epsilon(\tau) & =(1.0+2.66 \tau)^{-1} & & \text { (resonance lines), } \\
\epsilon(\tau) & =(1.0+2.66 \tau)^{-1}, & & \tau<3000 \\
& =\left[1.0+\left(4 \times 10^{4} \tau\right)^{1 / 2}\right]^{-1}, & & \tau \geq 3000
\end{array}\right\}
$$

(subordinate lines).

These were chosen to agree with the Monte Carlo calculations of Avery and House (1968; see also Hummer 1962; Capriotti 1965; Hubbard and Puetter 1984). The Fe II ion is treated as a five-level atom with rates and procedures described by CollinSouffrin et al. (1980) and Nussbaumer, Pettini, and Storey (1981). Trapping of resonance transitions of Mg II and Fe II, as well as subordinate transitions of Fe II, is also treated via the escape probability formalism. The helium triplets are treated as a five-level atom with rates and assumptions as in Feldman and MacAlpine (1978), with the exception that collisions between states that were not connected by an electric dipole allowed transition are given cross sections $\sim 2$ times lower, to be consistent with He spectra of planetary nebulae (see Kaler et al. 1976). Rates for collisional excitation from the ground state of hydrogen to the first two excited states are taken from Aggarwal (1983).

In addition to direct collisional excitation, we have also included continuum fluorescence for many lines. In the mean escape probability formalism, continuum pumping will proceed at a rate

$$
R_{l u}=\eta \epsilon(\tau) A_{u l}\left(N_{l}-N_{u}\right) g_{u} / g_{l} \mathrm{~s}^{-1},
$$

where $\eta$ is the continuum occupation number, $A_{u l}$ is the spontaneous emission rate, $g_{l}$ and $g_{u}$ are the statistical weights of the lower and upper states, and $\epsilon$ is the escape probability. This approach is used for pumping of Fe II levels through the ultraviolet transitions UV1, UV2, and UV3, and the optical [Fe $\mathrm{X}$ ] and [Fe XIV] coronal lines through the XUV resonance lines at $\sim 350 \AA$. The importance of continuum pumping in the former case has been stressed by Phillips (1979), and for the coronal lines in Seyfert galaxies, by Osterbrock (1969). We find that 
continuum fluorescence can be a significant excitation process in the models we consider.

Great care has been taken to incorporate fully the latest atomic cross sections and rates. The compendium by Mendoza (1983) has been used to update the entire collision strength and transition probability data base. Dielectronic recombination of $\mathrm{C}, \mathrm{N}$, and $\mathrm{O}$ through low-lying autoionizing levels has been included with the rate coefficients of Nussbaumer and Storey (1983). Secondary ionization by suprathermal electrons has been treated by combining the ionization efficiencies given by Shull (1979) and Bergeron and Collin-Souffrin (1973).

\section{c) Cosmic-Ray Interactions}

Synchrotron radio sources are usually modeled in terms of an interaction between a magnetic field and a relativistic gas with a typical energy per electron of a few hundred $\mathrm{MeV}$ (see Pacholczyk 1970); Longair 1981). The spectral index of the radio emission for radio-loud active galaxies is usually $\sim-0.7$, and this suggests that the electrons, which make the dominant contribution to synchrotron emission, have a density (per unit energy interval) given by $N_{e}(E) \sim E^{-2,4}$ (Kellermann 1966). The total electron density is sensitive to the lower bound of the energy distribution, which is typically of order $10-100 \mathrm{MeV}$, corresponding to relativistic factors of $\gamma \sim 10-100$ (cf. Lea and Holman 1978). We define a total relativistic electron density as

$$
N^{*}=\int_{E_{\min }}^{E_{\max }} N_{e}(E) d E,
$$

with the lower bound set to $E_{\min }=5 \mathrm{MeV}$, corresponding to a relativistic factor $\gamma \sim 10$. This density is only weakly sensitive to the upper limit $E_{\max }=10 \mathrm{GeV}$ because of the strong convergence of the electron density function. We ignore any possible cosmic-ray proton component, and present our results as a function of the total cosmic-ray electron density $N^{*}$. Since the Coulomb heating rate has no formal dependence on the cosmic-ray mass (in the relativistic limit), and the energy transfer rate has only a weak dependence on the relativistic factor $\left[\propto \log \left(\gamma^{2}\right)\right.$; see Jackson 1975], the effect of including a component of relativistic protons with the same energy distribution as the electrons would be to double the direct heating rate. (Subrelativistic protons will heat much more efficiently than relativistic electrons, but these particles may affect only the outer skin of the cloud, as discussed below.)

If the cosmic rays do indeed coexist with the optical emission-line regions, will they manage to penetrate and heat the cold gas? The line-emitting gas in the emission-line regions of active galaxies is generally thought to be in the form of small clumps or filaments. These filaments are optically thick to the low-energy ionizing continuum. As a result, the gas has a characteristic ionization and thermal structure; in general, both heating and ionization rates decrease with depth into the cloud, and radiative transfer effects are important.

In our discussion we assume that the cold gas is "optically thin" to the energetic electrons. Serious and fundamental uncertainties afflict detailed treatments of the penetration of energetic particles into cold gas, particularly if magnetic fields are present. For instance, if field lines are tangled near the cloud surface, then magnetic insulation effects can impede the flow of energetic particles into the gas. In the simplest case penetration is impeded only by ionization and heating losses resulting from two-body collisions. In this case, the ability to heat an entire cloud is determined by the range of a particle, or the column density of gas required to stop it (see Rossi 1952). Relativistic electrons have a range that is given to within $15 \%$ by (Berger and Seltzer 1965)

$$
R_{e}=10^{25}\left(\frac{E}{100 \mathrm{MeV}}\right)^{0.8} \text { particles } \mathrm{cm}^{-2}
$$

for a gas composed of neutral hydrogen. For comparison, the column density of the thickest cloud we model below is $\sim 10^{22}$ $\mathrm{cm}^{-2}$. (The range of a $100 \mathrm{MeV}$ electron in a fully ionized gas, in which bremsstrahlung and Coulomb losses are more important than ionization, would be some 10 times smaller, but BLR clouds typically have ionization fractions $\mathrm{H}^{0} / \mathrm{H}^{+} \sim 10$.)

The effective column density of gas seen by a cosmic-ray electron is increased by two factors, which together can act to impede the flow of energetic particles into the cloud. First, the gyroradius of the electron (if a magnetic field is present) is likely to be much smaller than the physical dimensions of the cloud. The effect is that the effective column density is increased by a factor of $\csc \alpha$, where $\alpha$ is the pitch angle of the electron. This effective column density is further increased by the collective effects discussed below. These effects increase the strength of the interactions between the two gases by large and uncertain amounts, and in turn decrease the range of the particles by similar amounts. These effects depend on details of the environment near the gas (particularly the geometry and magnetic field), but may decrease the range by factors $\sim 10-100$ if the relativistic and cold gases are in pressure equilibrium. The clouds we discuss below remain optically thin to $6 \mathrm{MeV}$ electrons for the larger factor, although these details are the most uncertain part of our calculation. Similarly, another fundamental uncertainty is posed by magnetic insulation effects, which can occur if field lines are tangled near the surface of a cloud.

A possible component of subrelativistic protons would probably not penetrate the entire cloud. For instance, a $100 \mathrm{MeV}$ proton has a range roughly 100 times smaller than an electron of the same energy, or roughly $10^{23} \mathrm{~cm}^{-2}$. As a result, protons with considerably lower energies would affect only the "skin" of the cloud. All of these effects introduce uncertainties that can be resolved only with a complete model of both the physical environment of the emission-line region and the cosmic-ray energy spectrum. For simplicity we consider only the relativistic electrons and assume that even the lowest energy $(\gamma \sim 10)$ cosmic rays are able to penetrate freely. As a result, the high-energy particle densities and heating rates are independent of depth into the cloud. Our treatment of cosmic-ray penetration is the best that can be done with the present computational resources, and seems adequate for exploratory work.

The relativistic particles both heat and ionize the gas. Our main concern here is for the rate with which energy is transferred to the cold gas (cf. Lea and Holman 1978; Ginzburg and Syrovatskii 1964). In the $\mathrm{H}^{+}$zone of the nebula the main interaction will be with free electrons. Kinetic energy is passed to the cold electrons at a rate

$$
\xi=N_{e} N^{*} 8.5 \times 10^{-19} \mathrm{ergs} \mathrm{cm}^{-3} \mathrm{~s}^{-1}
$$

by direct Coulomb interactions (cf. Jackson 1975; Cioffi 1984; Spitzer 1962; Ginzburg and Syrovatskii 1964; Pacholczyk 1970). Here $N_{e}$ is the thermal electron density, and we have integrated over the electron distribution given above.

In the $\mathrm{H}^{0}$ zone the main direct interaction between the two gases is through ionization of the cold gas. For large neutral 
fractions very little of the energy of secondary electrons goes into actually heating the gas (see Rossi 1952; Spitzer and Tomasko 1968); calculations show that secondary electrons have typical energies of $\sim 40 \mathrm{eV}$, and that there is roughly one ionization per $15 \mathrm{eV}$ deposited. Using the rates summarized by Ginzburg and Syrovatskii (1964 [the so-called Bethe-Bloch formula]), we find a neutral heating rate of

$$
\xi=N^{0} N^{*} 3.7 \times 10^{-20} \text { ergs } \mathrm{cm}^{-3} \mathrm{~s}^{-1}
$$

and an $\mathrm{H}^{0}$ ionization rate

$$
\Gamma^{*}=1.5 \times 10^{-8} N^{*} N^{0} \mathrm{~cm}^{-3} \mathrm{~s}^{-1}
$$

(see also Krolik, McKee, and Tarter 1981). This ionization rate has been scaled through Lotz's (1967) curves to include collisional ionization of heavy elements in our calculations.

Equation (6) includes only direct Coulomb interactions between the cosmic rays and the cold electrons, and does not include collective effects such as those discussed by Scott et al. (1980). As a result we underestimate the heating rate by factors that can be as large as $10^{5}$. Actually, it usually turns out that direct cosmic-ray ionization is less important as an ionization process than the heating term given by equation (6). This is because the rates for collisional ionization by thermal electrons are enhanced by the additional heat input due to the Coulomb interactions. These thermal ionizations occur near threshold, while cosmic-ray primary ionizations occur far from threshold where the ionization cross section is small. Because the dominant effect of including collective effects is to increase the factor in equation (6), which in turn dominates the other characteristics of the model, there will be a homology relation between models with different collective heating rates and cosmic-ray densities. We introduce a scale factor $\beta$, defined as the ratio of total heating to Coulomb heating, in order to account for this unmodeled heat input, and present our results as a function of the product $\beta N^{*}$. (For the case studied by Scott et al. 1980, $\beta=10^{5}$.)

The rates given by equations $(6)-(8)$ can be used to estimate those values of the electron and photon densities for which the relativistic particles will provide a significant source of heating and ionization. If every photoionization of hydrogen destroys a 1.5 ryd photon, the average for a typical power-law continuum, then 0.5 ryd of heat input will precede each recombination. The ratio of particles to photoelectric heat input will be

$$
\frac{\xi}{Q}=\frac{8.5 \times 10^{-19} \beta N^{*} N_{e}}{0.5 h v_{0} N_{e} N^{+} \alpha\left(T_{e}\right)}=3 \times 10^{5} \frac{\beta N^{*}}{N_{e}}
$$

where $\alpha\left(T_{e}\right)$ is the case B radiative recombination coefficient. Similarly, the high-energy particles provide an ionization source the importance of which relative to photoionization is given by

$$
\frac{\Gamma^{*}}{\Gamma}=\frac{1.5 \times 10^{8} N^{*} N^{0}}{\varphi \sigma_{0} N^{0}}=\frac{0.083}{U} \frac{N^{*}}{N_{e}} .
$$

In this expression $\varphi=Q(H) / 4 \pi r^{2}$ is the ionizing photon density $\left(\mathrm{s}^{-1} \mathrm{~cm}^{-2}\right) ; U$ is the ionization parameter, defined as $\varphi / N_{e} c ; r$ is the source-cloud separation; and we assume that one neutral-zone ionization occurs for each ionizing photon that enters the cloud. The last assumption is reasonable, since the photons that do enter the neutral zone are relatively hard and capable of producing many secondary ionizations.

Synchrotron emission requires the presence of a magnetic field in addition to the relativistic gas. If the magnetic field permeates the cool gas, then additional cooling by optically thin cyclotron emission can be significant under some circumstances. This process is related to the total cooling rate by (see Fabian, Pringle, and Rees 1976)

$$
\frac{\Lambda_{\text {cyclo }}}{\Lambda_{\text {tot }}}=\frac{N_{e}\left(B^{2} / 8 \pi\right) 2 \sigma_{T} c(V / c)^{2}}{0.5 h v_{0} \alpha\left(T_{e}\right) N_{e} N^{+}}=\frac{0.22 B^{2} T_{e}}{N_{e}} .
$$

This cooling mechanism is negligible for parameters of interest here $\left(B \sim 10^{-3}\right.$ gauss, $T \sim 10^{4} \mathrm{~K}, N_{e} \sim 10^{10} \mathrm{~cm}^{-3}$ for the BLR). We also assume that the prevalent magnetic field is not significantly enhanced within the gas cloud. Were such the case, the gyroradius of relativistic particles could be small enough to inhibit their flow into the cloud (i.e., the magnetic insulation effects alluded to above).

Finally, we note that, for comparison with radio observations that estimate the pressure due to relativistic electrons, the relationship between $N^{*}$ and this pressure is $N^{*}=1.0 \times 10^{5}$ $P_{\text {rel }}$, where we use the parameters given above, and assume that the pressure is due entirely to fully relativistic electrons in equipartition with the magnetic field. We also note a constraint, studied by Balbus and McKee (1982), of the stability of these clouds when $P_{\text {rel }}$ approaches the cold-gas pressure. Such clouds tend to be unstable to disruption by pressure gradients when they are supported entirely by nonthermal energetic particles.

\section{SPECIFIC MODELS}

\section{a) Broad-Line Region Clouds}

Baldwin's (1977) discovery of the so-called Ly $\alpha / \mathrm{H} \beta$ problem in the spectra of quasars has rejuvenated interest in photoionization models of these objects (see Canfield and Puetter 1980; Kwan and Krolik 1981; Weisheit, Shields, and Tarter 1981). It has long been known that the energy density of the relativistic particles within compact radio components is comparable to that of the BLR gas (Mathews 1974). Miley and Miller (1979) have established correlations between compact radio structure and Fe II emission, while Grandi and Osterbrock (1978) show that radio galaxies have steeper Balmer decrements and stronger $\mathrm{Fe}$ II emission than Seyfert galaxies. Considering a mixture of radio-loud and -quiet objects, Clavel and Joly (1984) found that Fe II emitters tended to have larger C III] $\lambda 1909 / C$ IV $\lambda 1549$ intensity ratios. Apparently, some aspects of the emission-line formation mechanism are somehow coupled to the radio properties of the nucleus.

Typically the compact radio lobes of radio-loud objects have relativistic particle densities of $1 \mathrm{~cm}^{-3}<N^{*}<10^{8.5} \mathrm{~cm}^{-3}$ (see Burbidge, Jones, and O'Dell 1974). Although these compact radio components occur at roughly the BLR radius, it is an open question whether the two gases actually mix. The only previous constraint on the cosmic-ray flux deduced from the emission-line spectrum was that of Baldwin et al. (1977). They set limits of $N^{*}<10^{6} \mathrm{~cm}^{-3}$ by considering the radiation damage suffered by each cloud and estimating the total exposure time.

We reconsider the "standard" model of Kwan and Krolik (1981) in detail here. As is usually the case for clouds ionized by a continuum with a significant X-ray component, this model is effectively matter-bounded. The original Kwan and Krolik model had a column density of $10^{23} \mathrm{~cm}^{-3}$, which was set by integrating until $\left.\mathrm{C}_{\mathrm{II}}\right] \lambda 2326$ reached its observed strength. This corresponded to a total linear thickness of $6.3 \times 10^{12} \mathrm{~cm}$. The larger part of this gas is fairly neutral; the hydrogen half- 

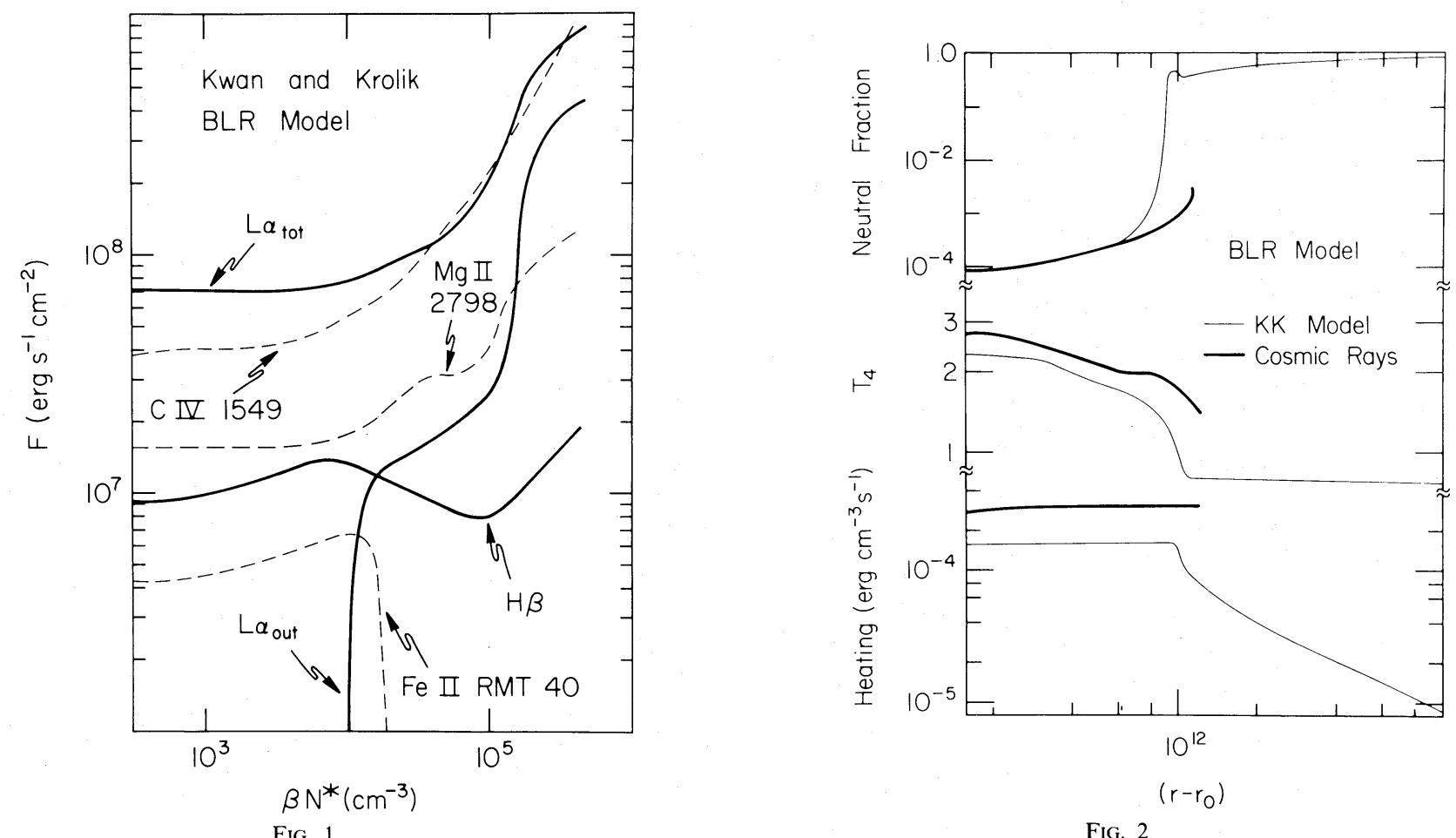

F1G. 1.-Clouds in broad-line region. This figure shows the effects of various densities of relativistic particles on the BLR cloud model of Kwan and Krolik (1981). The scale factor $\beta$ is the ratio of total to Coulomb heating, and can be as large as $10^{5}$. Intensities are expressed in terms of power radiated per square centimeter of cloud. The models have all parameters the same as the "standard" model of Kwan and Krolik (1981), with the exception of the cosmic-ray flux. The column density through the model was set by stopping radial integrations when the $\left.\mathrm{C}_{\mathrm{II}}\right] \lambda 2326 / \mathrm{C}_{\mathrm{IV}} \lambda 1549$ intensity ratio reached its observed value of 0.1 . Compact radio sources, which occur at roughly the radius of the broad-line region, typically have relativistic particle densities of $\sim 10 \mathrm{~cm}^{-3}-10^{8} \mathrm{~cm}^{-3}$.

Fig. 2. - Ionization structures. This figure compares the ionization and thermal structure of the Kwan and Krolik "standard" model (solid line) with the same model but with an added flux of cosmic rays of density $10^{4.5} \mathrm{~cm}^{-3}$. This density is near the logarithmic mean of the values determined for compact radio lobes of AGNs by Burbridge, Jones, and O'Dell (1974). Both models have column densities set by integrating through the cloud until the C II] $\lambda 2326 / C$ IV $\lambda 1549$ ratio reaches the observed value of 0.1 . This ratio is reached with a much lower column density $\left(1.01 \times 10^{22} \mathrm{~cm}^{-1}\right.$ as opposed to $10^{23} \mathrm{~cm}^{-1}$ for the Kwan and Krolik model) with the cosmic rays because of the additional heating the relativistic particles provide. The results of these two models are compared in Table 1.

neutral point occurs at a depth of only $1.0 \times 10^{12} \mathrm{~cm}$. It is the line-transfer effects that occur within the extended neutral zone that produce the anomalous $\mathrm{Ly} \alpha / \mathrm{H} \beta$ ratio.

We make the same assumptions as Kwan and Krolik (1981) in setting parameters of the model. The ionizing continuum is their broken power law, and the cloud has constant gas pressure and an inner hydrogen density of $N_{\mathrm{H}}=10^{9.6} \mathrm{~cm}^{-3}$. The chemical composition is nearly solar, with the exception of carbon, which is depleted by a factor of 2 . We use both their ionization parameter and their stopping criteria (e.g., radial integrations cease when the C II] $\lambda 2326 / C$ IV $\lambda 1549$ intensity ratio reaches 0.1 ). Our predictions are in good general agreement with those of Kwan and Krolik (1981) (for small $N^{*}$ ), despite the many changes in the atomic data base that have occurred since their calculations were performed.

Figure 1 shows some results of these calculations. These can be understood as the twofold consequences of increasing the heating and ionization rates through the addition of a component of high-energy particles. In any steady state model the total intensity of all emission lines produced at a particular point is set by the heating rate at that point. In the Kwan and Krolik (1981) model (see Fig. 2), a large portion of the total power radiated originates in the small $\mathrm{H}^{+}$"skin" on the inner boundary of the filament. This follows because the heating rate is largest where the continuum is relatively unextinguished. Under normal circumstances (e.g., negligible $N^{*}$ ), a very large extended neutral zone is required to produce appreciable emis- sion in lines from $\mathrm{Mg}^{+}, \mathrm{Fe}^{+}, \mathrm{C}^{+}$, and $\mathrm{H}^{0}$ because of the lower heating rate where the radiation field has been extinguished.

When the cosmic rays are first added, they affect this outer neutral zone more than inner regions, since this is where the original photoelectric heating and ionization rates were smallest. A consequence is that the heating rate and line emissivities in the "back" of the cloud increase, and the column density needed to produce the desired C II]/C IV ratio decreases. (Had we maintained constant column density rather than constant $\mathrm{C}$ II $/ \mathrm{C}$ IV line ratio, many lines originating in the neutral zone, particularly C II] $\lambda 2326$, would achieve prodigious strength.) The $\operatorname{Ly} \alpha / \mathrm{H} \beta$ ratio grows larger because of additional Ly $\alpha$ production, largely because the gas is growing hotter. The optical depths in nearly all lines grow smaller, and the absolute strength of $\mathrm{H} \beta$ changes in a complicated manner because of both the decreasing optical depth and the increasing temperature (see also Fig. 3 of Netzer 1975).

As $\beta N^{*}$ increases still further, the ionization and temperature continue to increase, and even some lines that are formed mainly in the $\mathrm{H}^{+}$skin (e.g., $\mathrm{C}$ IV 21549 ) strengthen relative to Ly $\alpha$. Some lines weaken (e.g., the Fe II lines) because they are no longer formed by dominant stages of ionization. Eventually the additional heating and ionization make the dominant contribution to the thermal and ionization equilibrium of the cloud, and a "nebular" solution is no longer found. As is typical of ionized gas, the cooling curve for these clouds reaches a peak in the neighborhood of $10^{5} \mathrm{~K}$ (see Dal- 
garno and McCray 1972). When the heating rate exceeds this maximum cooling, typically at a ratio of relativistic to cold electrons of $\beta N^{*} / N_{e}=10^{-4}$, the gas undergoes a thermal runaway and reaches relativistic temperatures. [This result is in good agreement with Krolik, McKee, and Tarter's (1981) estimate of the largest additional heat input consistent with a nebular solution.] This runaway actually sets a more stringent limit to $N^{*}\left(<\beta^{-1} 10^{5.5} \mathrm{~cm}^{-3}\right)$ than does the radiation-damage argument of Baldwin et al. (1977).

To consider the implications of these results for correlations between emission-line spectra and radio properties, we give results of two models in detail. In addition to the model with the original Kwan and Krolik (1981) parameters, Figure 2 shows a model with $\beta N^{*}=10^{4.5} \mathrm{~cm}^{-3}$, and Table 1 lists detailed predictions. In general, many of the optically thin intercombination lines strengthen relative to more nearly thermalized lines (such as $\mathrm{Ly} \alpha$ ) in response to the additional heat input. As was mentioned above, the $\mathrm{Fe}$ II emission has weakened considerably because very little $\mathrm{Fe}^{+}$is present in the model. This is mainly because the model with the cosmic rays was able to reproduce the desired C II] $\lambda 2326$ intensity with a relatively small column density, so that most iron is still doubly ionized at the back face of the cloud (the ionization potential of $\mathrm{C}^{+}$is $\sim 50 \%$ larger than that for $\mathrm{Fe}^{+}$). The calculations do not reproduce the steeper Balmer decrements observed in radioloud objects, which Osterbrock (1984) notes could be evidence for embedded dust, nor do they predict the larger C III]/C IV ratio in $\mathrm{Fe}$ II emitters.

An interesting prediction is that the Ly $\alpha$ line should become

TABLE 1

BLR CLOUD MODELS

\begin{tabular}{|c|c|c|}
\hline & $N^{*}=0$ & $\beta N^{*}=10^{4.5} \mathrm{~cm}^{-3}$ \\
\hline & $\begin{array}{l}L(\operatorname{Ly} \alpha)^{\mathrm{a}}=7.22(+7) \\
\tau_{\mathrm{Ly} \alpha}=5.26(+9) \\
\tau_{\mathrm{H} \alpha}=6.19(+4)\end{array}$ & $\begin{array}{l}L(\mathrm{Ly} \alpha)^{\mathrm{a}}=9.38(+7) \\
\tau_{\mathrm{Ly} \alpha}=7.73(+6) \\
\tau_{\mathrm{H} \alpha}=5620\end{array}$ \\
\hline Ly $\alpha \lambda 1216 \ldots \ldots \ldots \ldots \ldots$ & $1000^{\mathrm{b}}$ & $1000^{\mathrm{b}}$ \\
\hline $\mathrm{H} \alpha \lambda 6563 \ldots \ldots \ldots \ldots \ldots$ & 481 & 248 \\
\hline $\mathrm{H} \beta \lambda \lambda 4861 \ldots \ldots \ldots \ldots \ldots$ & 106 & 73 \\
\hline $\mathrm{He}$ I $\lambda 5876 \ldots$ & 11 & 16 \\
\hline He I $\lambda 10830$. & 56 & 65 \\
\hline He II $24686 \ldots \ldots \ldots \ldots \ldots \ldots$ & 3 & 3 \\
\hline $\mathrm{C}_{\text {II }} \lambda 2326$. & 41 & 127 \\
\hline C III $\lambda 1909 \ldots \ldots$ & 96 & 188 \\
\hline C III 2977 & 48 & 153 \\
\hline$C_{\text {IV }} \lambda 1549$ & 608 & 1115 \\
\hline $\mathrm{N}$ IV] $\lambda 1486$. & 53 & 97 \\
\hline $\mathrm{N} \vee \lambda 1240 \ldots \ldots \ldots \ldots \ldots$ & 48 & 87 \\
\hline $\mathrm{O} \mathrm{III}] \lambda 1666 \ldots$ & 149 & 302 \\
\hline $\left.\mathrm{O}_{\mathrm{IV}}\right] \lambda 1401 \ldots \ldots$ & 65 & 120 \\
\hline O vi $\lambda 1034 \ldots \ldots \ldots \ldots \ldots \ldots$ & 63 & 134 \\
\hline $\operatorname{Mg}$ II 22798 & 215 & 96 \\
\hline Fe II RMT $40^{c}$ & 51 & 2 \\
\hline Si III $\lambda 1895 \ldots \ldots \ldots \ldots \ldots$ & 25 & 550 \\
\hline Si iv $\lambda 1397 \ldots$. & 45 & 125 \\
\hline $\mathrm{S}_{\text {IV }} \lambda 1406 \ldots \ldots \ldots \ldots \ldots$ & 7 & 25 \\
\hline
\end{tabular}

${ }^{\text {a }}$ In units of ergs $\mathrm{s}^{-1} \mathrm{~cm}^{-2}$.

b Relative intensity.

${ }^{c}$ Revised Multiplet Table. more nearly symmetric as $N^{*}$ increases. One of the most promising models for the origin of BLR clouds is the radiatively accelerated outflowing wind as developed by Blumenthal and Mathews $(1975,1979)$. The greatest shortcoming of this model is its prediction of an extremely asymmetric Ly $\alpha$ profile. This follows because Ly $\alpha$ is almost entirely formed in the $\mathrm{H}^{+}$skin on the inner side of the BLR cloud, and hence is preferentially radiated in the direction of the central object (Ferland, Netzer, and Shields 1979). This is in sharp contrast to the symmetric Ly $\alpha$ usually observed (cf. Baldwin and Netzer 1978). As Figure 1 shows, significant emission in the outward direction can be produced, largely because of enhanced Ly $\alpha$ emission from the back of the cloud. This happy circumstance occurs, however, only when $\beta N^{*}$ is larger $\left(>10^{5} \mathrm{~cm}^{-3}\right)$ than would actually be allowed, considering other line ratios such as $\operatorname{Ly} \alpha / \mathrm{H} \beta$. (One other result of the fact that all hydrogen lines are emitted from both sides of the cloud is that convergence of the entire model was slow. Typically four to six iterations were needed.)

Interactions between the radio components and the BLR clouds could also explain the absence of emission lines in BL Lac objects. Models proposed so far to account for this lack include the absence of a sufficient amount of gas to produce the lines (Shields 1978), an alignment with the relativistic jets pointed toward the observer (Blandford and Rees 1978), and an intrinsic inability for emission-line clouds to form (Guilbert, Fabian, and McCray 1983). We point out another possibility, namely, that relativistic particles are mixed with the BLR gas and cause it to emit a negligible amount of optical radiation. Figure 1 shows that if $\beta N^{*} / N>10^{-4.4}$, the gas will undergo a thermal runaway. Such gas would produce little optical emission.

\section{b) Narrow-Line Region}

Osterbrock (1983) speculates that the relativistic plasma within radio jets may explain the high temperature often deduced from the $[\mathrm{O}$ III $] \lambda 5007 / \lambda 4363$ ratio. Heckman and Balick (1979) find temperatures in excess of $2 \times 10^{4} \mathrm{~K}$ for NLRs in their sample of broad-line objects, while Cohen and Osterbrock (1981) find even higher temperatures for some narrow-line radio galaxies. These temperatures are surprising, since photoionization generally produces temperatures close to $10^{4} \mathrm{~K}$ (see Osterbrock 1974).

We model a typical NLR as a set of clouds of constant density $\left(N=10^{3} \mathrm{~cm}^{-3}\right)$ and an ionization parameter (defined as the ratio of ionizing photons to free electrons at the inner face of the cloud) of $U=10^{-2}$ (Ferland 1981). This singlecomponent model is an obvious oversimplification (see Netzer 1982; Péquignot 1984), but the results should be of heuristic value. The clouds are ionized by a broken power-law continuum, with a steep continuum (spectral index $=-1.5$ ) from the optical to $400 \mathrm{eV}$ and a shallower (spectral index $=-0.5$ ) continuum extending to $100 \mathrm{keV}$. Metal abundances are reduced to 0.5 dex below solar as in Ferland and Netzer (1983). In these models we stop radial integrations when the $\left[\mathrm{O}_{\mathrm{I}}\right] \mathrm{\lambda 6300/}$ [O III] $\lambda 5007$ ratio reaches its observed value of 0.05 .

Results of our calculations are shown in Figure 3. The cosmic rays first affect the emission-line spectrum when the density reaches $\beta N^{*} \sim 10^{-4} \mathrm{~cm}^{-3}$. As was also the case for the BLR models considered above, the first lines to be affected are those produced in the neutral regions where the photoelectric heating rates are smallest. Both the absolute intensity of $\mathrm{H} \alpha$ and the Balmer decrement $\mathrm{H} \alpha / \mathrm{H} \beta$ increase with $\beta N^{*}$ because of the enhanced collisional excitation rates caused by the 


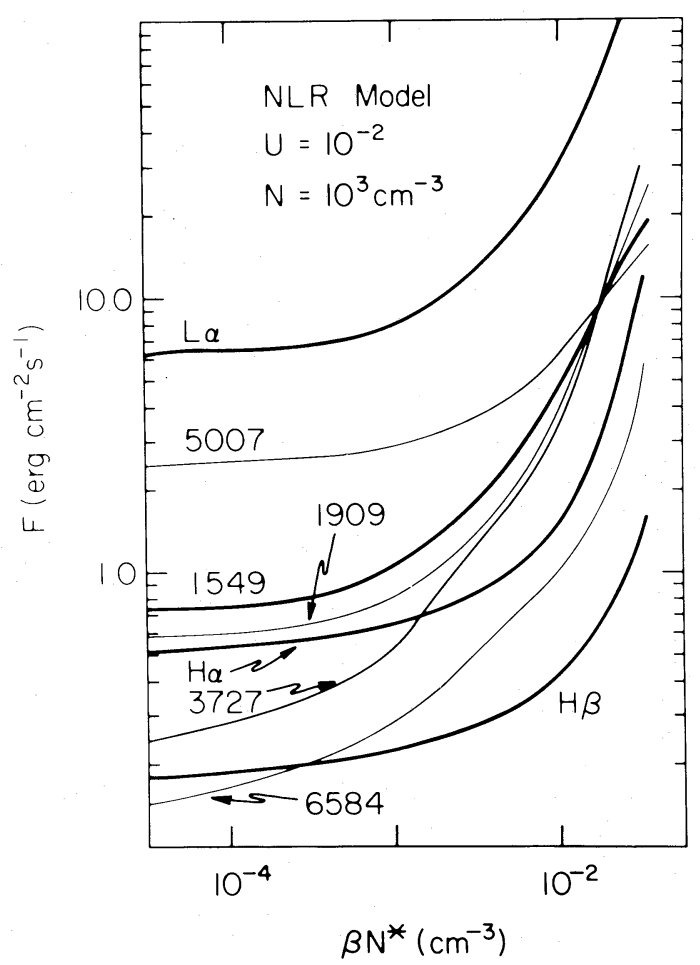

Fig. 3.-Models of narrow-line region. This figure shows the results of an added flux of cosmic rays on a model of a typical narrow-line region. The models had a variable column density, which was chosen to maintain a constant $\lambda 6300 / \lambda 5007$ intensity ratio of 0.05 , near the observed value.

higher temperature. Again, as was true for the BLR, lines formed in the ionized regions of the cloud are affected relatively less, a consequence of the larger photoelectric heating rates there. Eventually the additional heating is large enough to keep hydrogen collisionally ionized, and little [O I] emission is ever formed $\left(\beta N^{*}>10^{-1} \mathrm{~cm}^{-3}\right)$.

Table 2 compares some predictions of models with and without the cosmic-ray contribution. The cosmic-ray density is $\beta N^{*}=10^{-1.5} \mathrm{~cm}^{-3}$, the largest value consistent with the presence of significant [O $\mathrm{O}$ ] emission. Our calculations support the view that the relativistic gas associated with the radio lobes could produce the high [O III] temperatures in some cases. The ratio $\lambda 5007 / \lambda 4363$ decreases by a factor of 2 , indicating a larger electron temperature, when the cosmic rays are added. The temperature of the $\mathrm{O}^{++}$zone doubles from $\sim 1.4 \times 10^{4} \mathrm{~K}$ for negligible $\beta N^{*}$ to $\sim 2.9 \times 10^{4} \mathrm{~K}$ for $\beta N^{*}=10^{-1.5} \mathrm{~cm}^{-3}$.

The cosmic rays cause a particular enhancement of lines from both neutral and transition regions of the nebula, again because the original heating rate was lowest there. In particular, this causes the spectrum to resemble that of a lowionization nuclear emission-line region in many ways (see Heckman 1980). A simple analysis of the emission spectrum with the additional heat input would deduce sharply enhanced heavy-element abundances because of the strengthening of the forbidden lines.

\section{c) Optical Emission from Extended Radio Lobes}

Careful scrutiny of deep optical plates taken of regions within the extended radio lobes of AGNs often reveals small filaments or condensations (cf. Graham and Price 1981; Miley et al. 1981; Heckman et al. 1982; Burns, Feigelson, and Schreier 1983; Brodie, Königl, and Bowyer 1983). Several mechanisms have been proposed to explain the origin of this emission, ranging from shocks to embedded star formation, and we wish to consider another. Could these filaments be powered by the relativistic gas that surrounds them?

We model filament 5 of Graham and Price's (1981) paper on Cen $\mathrm{A}$ in detail. This is a faint condensation roughly $22^{\prime}$ away from the nucleus along the northeast radio axis, near the center of the middle radio lobe. In contrast to the cases considered above, there can be little doubt that the optical filaments are in fact surrounded by the radio emitting plasma. Other properties, such as the X-ray luminosity, distance, and emission measure, are well determined; this object is an excellent candidate for detailed study.

We assume constant gas pressure and an inner gas density of 0.1 hydrogen atom per cubic centimeter, resulting in an average density of $N \sim 0.2 \mathrm{~cm}^{-3}$, as deduced by Graham and Price (1981). (Note that the cooling time for a gas of this density is $\sim 2000$ years, so that a heating source is clearly needed to support the observed emission.) For an assumed distance of 3.3 Mpc to Cen A (de Vaucouleurs 1979), the component lies $6.3 \times 10^{22} \mathrm{~cm}$ from the nucleus and has a thickness of $3.2 \times 10^{21} \mathrm{~cm}$. We also assume a uniform heavy-element depletion factor of 0.1 .

TABLE 2

NLR MODELS

\begin{tabular}{|c|c|c|}
\hline & $N^{*}=0$ & $\beta N^{*}=10^{-1.5} \mathrm{~cm}^{-3}$ \\
\hline & $\begin{array}{c}L(\mathrm{H} \beta)^{\mathrm{a}}=0.181 \\
N_{\mathbf{H}}^{\mathrm{b}}=2.24(+22)\end{array}$ & $\begin{array}{c}L(\mathrm{H} \beta)^{\mathrm{a}}=1.42 \\
N_{\mathrm{H}}^{\mathrm{b}}=\mathrm{R} 2.53(+22)\end{array}$ \\
\hline$\overline{\operatorname{Ly} \alpha \lambda 1216}$. & $3431^{\mathrm{c}}$ & $23600^{c}$ \\
\hline H $\beta \lambda 4861 \ldots \ldots \ldots$ & 100 & 100 \\
\hline $\mathrm{H} \alpha \lambda 6563 \ldots \ldots \ldots \ldots \ldots \ldots$ & 279 & 787 \\
\hline $2 v \ldots \ldots \ldots \ldots$ & 1052 & 12700 \\
\hline $\mathrm{He}$ I $\lambda 5876$ & 12 & 14 \\
\hline He I $\lambda 10830 \ldots$ & 81 & 98 \\
\hline He II $\lambda 4686 \ldots \ldots$ & 25 & 10 \\
\hline $\left.\mathrm{C}_{\mathrm{II}}\right] \lambda 2326 \ldots$ & 55 & 668 \\
\hline C III $\lambda 977 \ldots$ & 25 & 125 \\
\hline C III] $\lambda 1909$. & 320 & 1582 \\
\hline C IV $\lambda 1549 \ldots$ & 399 & 1204 \\
\hline$[\mathrm{N} \mathrm{II}] \lambda 6584$ & 78 & 387 \\
\hline N IV $] \lambda 1486 \ldots \ldots \ldots \ldots \ldots$ & 16 & 88 \\
\hline $\mathrm{N} v \lambda 1240 \ldots \ldots \ldots \ldots \ldots$ & 8 & 28 \\
\hline$\left[\mathrm{O}_{\mathrm{I}}\right] \lambda 6300$. & 70 & 59 \\
\hline$\left[\begin{array}{ll}\mathrm{II} & \lambda 3727 \\
& \end{array}\right.$ & 136 & 2036 \\
\hline$\left[\mathrm{O}_{\mathrm{II}}\right] \lambda 7325$. & 5 & 96 \\
\hline$[\mathrm{O}$ III $] \lambda 5007$. & 1365 & 1172 \\
\hline$[\mathrm{O} \mathrm{III}] \lambda 4363$. & 24 & 65 \\
\hline$[\mathrm{Ne} \mathrm{HI}] \lambda 3869 \ldots \ldots \ldots \ldots$ & 105 & 142 \\
\hline Mg $1122798 \ldots$ & 73 & 953 \\
\hline $\mathrm{Fe}$ II RMT $40^{\mathrm{d}}$ & 3 & 47 \\
\hline Si III $\lambda 1397$......................... & 34 & 168 \\
\hline$\left[\mathrm{S}_{\mathrm{II}}\right] \lambda 6731$. & 165 & 898 \\
\hline$\left[\mathrm{S}_{\text {II] }} \lambda 4074\right.$. & 7 & 104 \\
\hline$\left[\mathrm{S}_{\text {III }}\right] \lambda 9532 \ldots$ & 189 & 115 \\
\hline$[$ Ar IV] $\lambda 4740$. & 6 & 10 \\
\hline
\end{tabular}

${ }^{a}$ In units of ergs $\mathrm{s}^{-1} \mathrm{~cm}^{-2}$.

${ }^{b}$ In units of $\mathrm{cm}^{-2}$.

${ }^{\mathrm{c}}$ Relative intensity.

${ }^{\mathrm{d}}$ Revised Multiplet Table. 


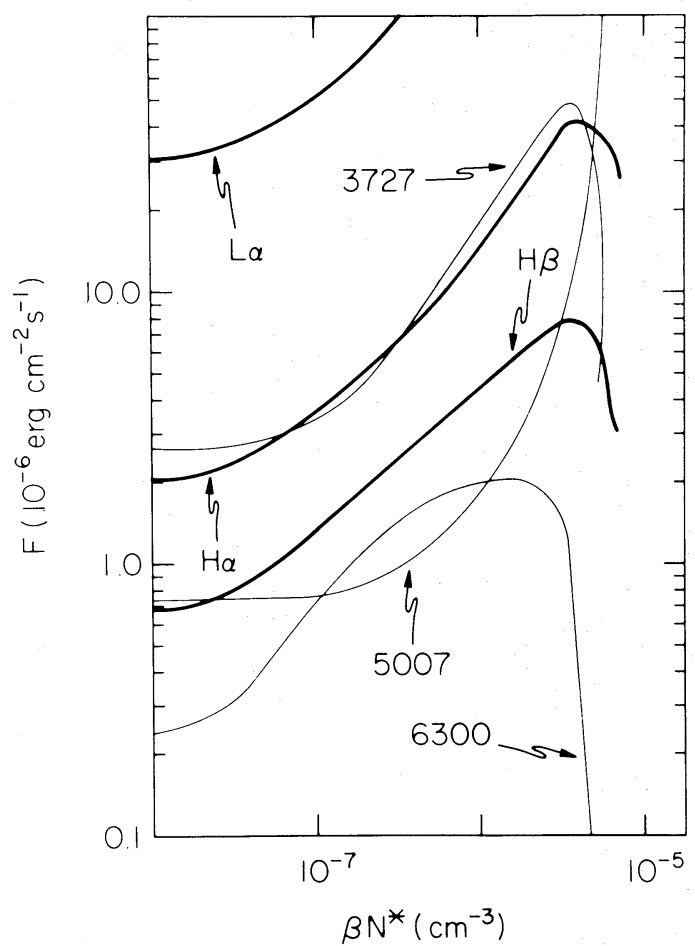

FIG. 4.-Extended optical emission. This figure shows the effects of a flux of cosmic rays upon a low-density filament similar to those typically found in radio lobes of active nuclei. Parameters were chosen to be similar to those of filament 5 of Cen A as measured by Graham and Price (1981). This emission component lies $\approx 22^{\prime}$ away from the nucleus, near the center of the northeast middle radio lobe. This model has constant gas pressure, a uniform metal depletion factor of 0.1 , and an inner density of $0.1 \mathrm{~cm}^{-3}$, and is ionized by both a power-law continuum (corresponding to an ionization parameter $U=10^{-3.5}$ ) and the cosmic rays. The cloud is only partially ionized in the models with small cosmic-ray densities, and the level of ionization increases until the thermal runaway occurs for $\beta N^{*}=10^{-5}$.
We include the effects of both the ionizing radiation from the nucleus and the surrounding cosmic rays. A broken power-law continuum with an X-ray component characterized by a spectral index of -0.7 , and the steeper index of -1.5 for the optical, joining at $400 \mathrm{eV}$, is used. We normalize this continuum to match the $2-6 \mathrm{keV}$ flux of $\sim 5 \times 10^{-10} \mathrm{ergs} \mathrm{cm}^{-2} \mathrm{~s}^{-1}$ reported by Feigelson et al. (1981). The ionization parameter corresponding to this distance, luminosity, and density is $U=10^{-3.5}$, too small to account for the observed ionization and luminosity (as Graham and Price 1981 point out).

Figure 4 shows the results of varying the cosmic-ray density between the values where the first effects occur and the upper limit, above which no nebular solution is found. Table 3 compares the model predictions (with the ionizing radiation from the nucleus alone, and also with both this radiation and a cosmic-ray density of $\beta N^{*}=10^{-5.65} \mathrm{~cm}^{-3}$ ) with the Graham and Price (1981) observations. Again, the main effect of adding the cosmic rays is to increase the total luminosity of the lines by adding more heat and ionization. The $\mathrm{H} \beta$ luminosity increases by an order of magnitude, reflecting the fact that the filament is now fully ionized (the model without the cosmic rays was only partially ionized). The relative line strengths do not change greatly, although there is a tendency for a higher level of ionization because of larger collisional ionization rates.

The fact that a contribution from cosmic rays is required to reproduce the observed emission-line luminosity makes the strongest case for the fact that the interactions we have considered are are actually taking place. Although we know of no measurements of the cosmic-ray density or pressure for the radio lobes surrounding the emission-line region we consider, we can estimate the cosmic-ray density in the inner jet (a separation of $4^{\prime}$ from the nucleus, $N^{*}=10^{-5.9} \mathrm{~cm}^{-3}$ ) by combining the estimate of the pressure within this jet given by Burns, Feigelson, and Schreier (1983) with the relationship between gas pressure and density given in $\S$ II. $N^{*}$ is probably somewhat lower than this in the region we consider, which is nearly a factor of 5 more distant from the nucleus. Considering the wide range of possible values for collective heating and $\beta$,

TABLE 3

EXTENDED EMISSION REGIONS

\begin{tabular}{|c|c|c|c|}
\hline & OBSERVED & $N^{*}=0$ & $\beta N^{*}=10^{-5.65}$ \\
\hline LiNE & $F(\mathrm{H} \beta)^{\mathrm{a}} \sim 3$ & $F(\mathrm{H} \beta)^{\mathrm{a}}=0.12$ & $F(\mathrm{H} \beta)^{\mathrm{a}}=1.3$ \\
\hline $\operatorname{Ly} \alpha \lambda 1216$ & . & $4770^{b}$ & $11650^{b}$ \\
\hline $\mathrm{H} \mathrm{I}, 2 v \ldots \ldots \ldots$ & & 1860 & 5440 \\
\hline $\mathrm{H} \propto \lambda 6563$ & $330^{\mathrm{b}}$ & 299 & 433 \\
\hline $\mathrm{H} \beta \lambda 4861 \ldots$ & 100 & 100 & 100 \\
\hline $\left.\mathrm{C}_{\mathrm{II}}\right] \lambda 2326 \ldots$ & $\ldots$ & 81 & 140 \\
\hline C III] $\lambda 1909 .$. & $\cdots$ & 43 & 60 \\
\hline$\left[\mathrm{N}_{\mathrm{I}}\right] \lambda 25199$ & & 11 & 11 \\
\hline$[\mathrm{N} \mathrm{II}] \lambda 6584$ & 90 & 75 & 94 \\
\hline$\left[\mathrm{O}_{\mathrm{I}}\right] \lambda 26300$ & & 36 & 29 \\
\hline$\left[\begin{array}{lll}\mathrm{O} & \mathrm{II}\end{array}\right] \lambda 3727$ & 670 & 416 & 563 \\
\hline$[\mathrm{O}$ III $] \lambda 5007 \ldots \ldots \ldots \ldots \ldots$ & 30 & 115 & 59 \\
\hline$[\mathrm{Ne} \mathrm{III}] \lambda 3869$ & $\ldots$ & 46 & 31 \\
\hline $\mathrm{Mg}_{\text {II }} \lambda 2798$. & & 57 & 120 \\
\hline$[\mathrm{S}$ II $] \lambda 6731 \ldots \ldots \ldots \ldots \ldots$ & 100 & 212 & 278 \\
\hline
\end{tabular}

a In units of $10^{-17} \mathrm{ergs} \mathrm{s}^{-1} \mathrm{~cm}^{-2} \operatorname{arcsec}^{-2}$.

${ }^{b}$ Relative to $\mathrm{H} \beta$. 
we consider this measurement to be in adequate agreement with our model.

\section{DISCUSSION}

The calculations presented here have shown that the emission-line spectra from regions on all distance scales in AGNs would be affected by the addition of a component of relativistic particles with densities similar to those within the radio lobes found on the same distance scale. The main effect, for small $\beta N^{*}$, is to increase the strength of lines from species with low ionization potentials. Typically, the effects first become important for $N^{*} / N_{e}=10^{-7} / \beta$ and increase until, for $N^{*} / N_{e}=10^{-4.4} / \beta$, no nebular solution is found. It is important to bear in mind that we have included only heating due to direct Coulomb interactions between cosmic rays and cold electrons. The scale factor $\beta$ reflects that fact that our results are conservative underestimates of the actual effects. Collective effects, which increase the heating rate by a factor $\beta$, have not been included and will enhance the heating rates by many orders of magnitude.

Our original goal in examining this problem was to determine whether some properties of AGNs could be explained through the addition of a flux of high-energy particles. We have discussed several aspects of this question above: the causes of some spectral differences between radio-loud and radio-quiet AGNs, production of symmetric Ly $\alpha$ lines in an outflowing wind, quenching of BLR emission in BL Lac objects, the establishment of higher than expected [O III] temperatures in narrow-line regions of some AGNs, and, finally, cosmic rays as the energy source for the optical filaments within extended radio lobes. In the last case a conclusive argument for an interaction between cosmic rays and the cold gas can be made.

In principle it should be possible to decide whether the processes we have considered are actually taking place in other regions, by comparing the predicted and observed emissionline spectra. Unfortunately the differences between nebulae with and without this extra heating and ionization are not extreme, and it would be difficult to determine from the emission-line spectrum alone whether the additional com- ponent of high-energy particles was present. For the case of the BLR and NLR models, the addition of the cosmic rays actually reduces the agreement between theory and observation for some line ratios, while bringing other line ratios into better agreement. It is important to remember that our goal here was only to determine whether such heat input could be important for several standard cases; in particular, we have not reexamined all of parameter space to reoptimize model fits. The "standard" parameters we have used in considering the broadand narrow-line regions (such as density, composition, and ionization parameter) were in turn set by optimizing model fits. A different set of parameters would have been chosen if the relativistic had been included in the parameter optimization. Nonetheless, it seems difficult to escape the conclusion that the additional flux of cosmic rays does little to help explain the Grandi and Osterbrock (1978) correlation.

To summarize our results, we would say that the relativistic gas that produces the nonthermal radio emission would certainly alter the emission-line spectrum if the two gases mix. We consider this, together with the models of the extended emission-line regions, to be the most important result of this paper. Our intention is more in keeping with the philosophy of Daltabuit, MacAlpine, and Cox (1978) in their examination of the effects of shocks on a typical BLR cloud; these processes could well occur and should be kept in mind. Whether the two gases do in fact mix could perhaps best be determined by careful high-resolution mapping of the emission-line regions by the Space Telescope, followed by a detailed comparison with VLBI data.

G. J. F. acknowledges the hospitality of Lick Observatory and the Laboratory for High Energy Astrophysics, where part of this work was carried out. The support of the NSF through grant AST 83-05094 and informative conversations with Moshe Elitzur, William Mathews, Richard McCray, Donald Osterbrock, Richard Puetter, and Gregory Shields are also appreciated. We are grateful to the anonymous referee for his help in bringing some of our ideas into focus, and for stressing the complexities of cosmic-ray interactions with matter.

\section{APPENDIX A}

\section{A CRAB NEBULA FILAMENT}

Henry and MacAlpine (1982) have performed extensivē predictions of emission-line spectra for several filaments in the Crab Nebula. We focus attention on one set of models, those for filament 5 of Fesen and Krishner (1982). As was the case for the optical emitters within radio lobes, it is clear that the filaments are surrounded by relativistic electrons, although it is not clear whether an embedded magnetic field might shield the cold gas from the cosmic rays. Except for the addition of a variable component of relativistic electrons, the models we have run are identical with model 5.1 of Henry and MacAlpine (1982). Basically, it is a constant-pressure, plane-parallel cloud with an inner hydrogen density of $10^{3.3} \mathrm{~cm}^{-3}$, a chemical composition with enhanced $\mathrm{He}$ and $\mathrm{C}$, solar $\mathrm{Mg}, \mathrm{Si}, \mathrm{S}, \mathrm{Ar}$, and $\mathrm{Fe}$, and depleted $\mathrm{N}, \mathrm{O}$, and $\mathrm{Ne}$, ionized by a power-law continuum extending to $100 \mathrm{keV}$. The slab is effectively matter-bounded (optically thin to hard X-rays), and has a total thickness of $2 \times 10^{17} \mathrm{~cm}$, although the hydrogen half-neutral point occurs at a depth of $6.7 \times 10^{16} \mathrm{~cm}$. Tests show good overall agreement between Henry and MacAlpine's calculations and ours for small $N^{*}$.

Figure 5 shows the results of our calculations. We vary the relativistic particle density between $\beta N^{*}=10^{-4} \mathrm{~cm}^{-3}$, where the first effects occur, and $\beta N^{*}=10^{-1} \mathrm{~cm}^{-3}$, above which no nebular solution is found. Two solutions $\left(T \sim 10^{4} \mathrm{~K}, T \sim 10^{6}\right.$ $\mathrm{K})$ can exist for values of $\beta N^{*}$ in the range $10^{-2}-10^{-1} \mathrm{~cm}^{-3}$, a situation also found in some models of photoionized gas near an X-ray source (see Hatchett, Buff, and McCray 1976); we plot the low-temperature solution. 


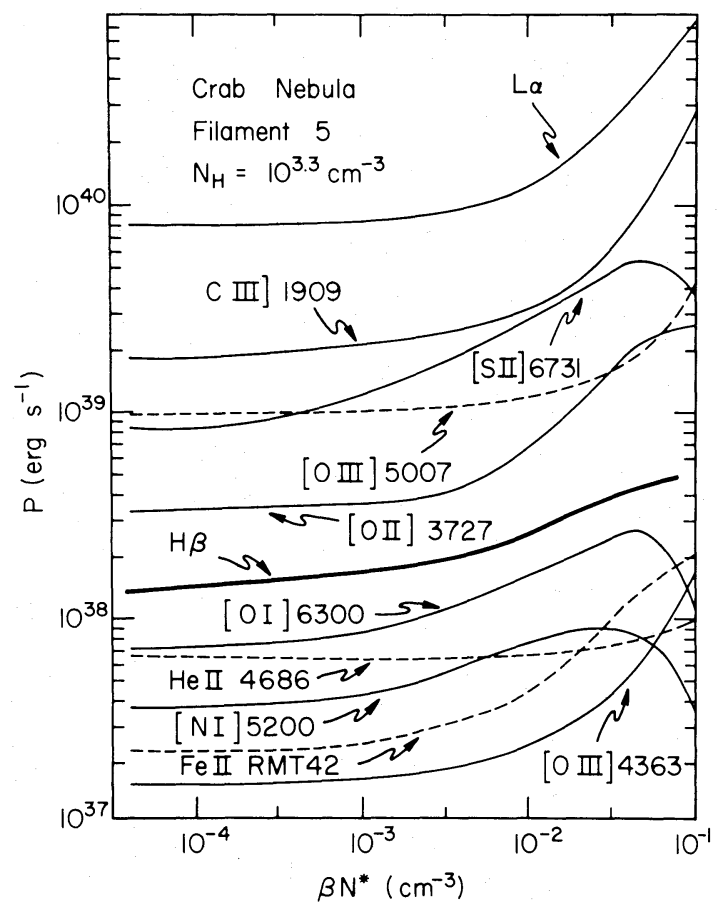

FIG. 5.-Crab Nebula filaments. This figure shows the effects of a flux of relativistic particles on model 5.1 of Henry and MacAlpine (1982). Intensities are expressed in total power radiated into $4 \pi$ steradians by a filament fully covering the source of ionizing radiation.

The total particle density within the Crab Nebula itself is difficult to estimate, but Faraday rotation studies indicate $N<10^{-2} \mathrm{~cm}^{-3}$ (Wilson 1974). Direct integration of the spectrum of the Crab Nebula between $10^{8}$ and $10^{20} \mathrm{~Hz}$ indicates
$N \sim 10^{-6}-10^{-5} \mathrm{~cm}^{-3}$, depending on the magnetic field. The cosmic-ray density is probably too small to have any observable effect, even if the gases do mix, unless $\beta>10^{2}$.

\section{APPENDIX B}

\section{HOW RELIABLE ARE PHOTOIONIZATION CALCULATIONS?}

In a series of papers, the most recent of which is that of Hubbard and Puetter (1984), Puetter and coworkers have questioned many of the basic premises of the type of calculation we have performed. Although this work has resulted in some substantial improvements in treatment of some aspects of line transfer, particularly the form of the escape probability function used, the series has also generated some confusion because of the radically different nature of the models. In particular, until recently, all of their calculations assumed constant electron temperature. Their most recent calculation performs the thermal balance but treats only hydrogen. The fact that their treatment of the line-transfer process is more realistic than ours, while at the same time they neglect the presence of the heavy elements entirely and use an oversimplified continuum, raises an important question. Do their calculations disagree with those of Kwan and Krolik (1981), with whom we find good agreement, because of sophistication or because of oversimplification? It is this question that we address here.

We have performed a set of calculations in which the abundances of all heavy elements are reduced by a factor of $10^{-5}$. We model the two cases considered by Hubbard and Puetter (1984) in detail, e.g., a lower density model with typical BLR parameters, as well as their high-density model. We treat the hydrogen line transfer with escape probabilities, and iterate on the solution while treating the diffuse fields with the so-called lambda-iteration procedure. Convergence was fairly rapid, typically after only three iterations. Our results, together with those of Hubbard and Puetter (1984), are summarized in Table 4. The agreement, particularly for the lower density model, is quite good $(\sim 5 \%)$. The poorer agreement in the high-density model, which has a much lower ionization parameter and hence larger neutral fraction, is largely due to the fact that Hubbard and Puetter neglect secondary ionization by suprathermal electrons in neutral regions (see Bergeron and CollinSouffrin 1973).

The excellent agreement between our current predictions and published thermal equilibrium models was commented on

TABLE 4

MODEL COMPARISONS

\begin{tabular}{|c|c|c|c|c|}
\hline \multirow[b]{2}{*}{ RATIO } & \multicolumn{2}{|c|}{$\begin{array}{c}P=0.0207 \\
\text { dynes } \mathrm{cm}^{-2}\end{array}$} & \multicolumn{2}{|c|}{$\begin{array}{c}P=0.414 \\
\text { dynes } \mathrm{cm}^{-2}\end{array}$} \\
\hline & $\mathrm{HP}^{\mathrm{a}}$ & Cloudy & $\mathrm{HP}^{\mathrm{a}}$ & Cloudy \\
\hline $\mathrm{H} \alpha / \mathrm{H} \beta$ & 8.5 & 8.88 & 2.5 & 3.23 \\
\hline $\operatorname{Ly} \alpha / \mathrm{H} \alpha \ldots \ldots \ldots \ldots$ & 5.5 & 5.94 & 1.1 & 1.63 \\
\hline $\mathrm{Bac} / \mathrm{H} \alpha \ldots \ldots \ldots$ & 0.35 & 0.37 & 0.13 & 0.27 \\
\hline $\operatorname{Pa} \alpha / \mathrm{H} \alpha \ldots \ldots \ldots$ & 0.07 & 0.073 & 0.1 & 0.09 \\
\hline
\end{tabular}

${ }^{a}$ Hubbard and Puetter model. 
above. The fact that we again find good agreement with the Hubbard and Puetter (1984) calculations (in particular for the model with standard BLR parameters) is not surprising. In all these cases, it is important to remember the point stressed by Davidson $(1973,1977)$ : no energy-conserving calculation can go far wrong. This is because the strength of the strongest emission lines at a given point in a model is simply set by the heating rate at that point. This, in turn, is actually set by the shape of the continuum and the recombination coefficient, each fairly simple and uncontroversial. In fact, tests show that there actually has been fairly little change in the predictions of such models as those of Davidson and Netzer (1979) when the same assumptions concerning the ionizing continuum and thickness of the cloud are made. This is particularly true for strong lines; their intensities cannot change greatly in an energy-conserving model.

Why, then, do the Hubbard and Puetter (1984) calculations disagree with those of Kwan and Krolik (1981) (as well as with our present results for the case of nebulae with heavy elements)? Tests show that the biggest differences are attributable to differences in the continuum shape and their neglect of both heavy-element opacities and cooling. The first two control the heating rate at great depths into the cloud (where the Balmer lines are formed), while the latter affects the thermal solution throughout.

The most important result of this comparison is the fact that a computationally expedient technique, the mean escape probability formalism, is in excellent agreement with the more sophisticated procedures followed by Hubbard and Puetter (1984), when the same assumptions concerning ionizing continuum, metallicity, and the form of the escape probability function are made. Modern techniques for computing emergent spectra from photoionized nebulae are both reliable and consistent.

\section{REFERENCES}

Aggarwal, K. M. 1983, M.N.R.A.S., 202, 15P.

Aldrovandi, S. M. V., and Péquignot, D. 1973, Astr. Ap., 26, 33.

Avery, L. W., and House, L. L. 1968, Ap. J., 152, 493.

Balbus, S., and McKee, C. 1982, Ap. J., 252, 529.

Baldwin, J. A. 1977, M.N.R.A.S., 178, 67P.

Baldwin, J., Boksenberg, A., Burbidge, G., Carswell, R., Cowsik, R., Perry, J., and Wolfe, A. 1977, Astr. Ap., 61, 165

Baldwin, J., and Netzer, H. 1978, $A p . J ., 226,1$.

Berger, M. J., and Seltzer, S. M. 1965, NASA SP-3012.

Bergeron, J., and Collin-Souffrin, S. 1973, Astr. Ap., 25, 1

Blandford, R. D., and Rees, M. J. 1978, in Pittsburgh Conference on BL Lac

Objects, ed. A. M. Wolfe (Pittsburgh: University of Pittsburgh), p. 123.

Blumenthal, G., and Mathews, W. 1975, Ap. J., 198, 517.

Blumentha, Ap. J., 233, 479.

Brodie, J., Königl, A., and Bowyer, S. 1983, Ap. J., 273, 154

Burbidge, G. R., Jones, T. W., and O'Dell, S. L. 1974, Ap. J., 193, 43.

Burns, J O Feigelson, E. D and Schreier, E. J. 1983, Ap. J, 273, 128

Canfield, R., and Puetter, R. 1980, Ap. J. (Letters), 236, L7.

Capriotti, E. R. 1965, Ap.J., 142, 1101.

Cioffi, D. F. 1984, preprint.

Clavel, J., and Joly, M. 1984, Astr. Ap.,131, 87.

Cohen, R. D., and Osterbrock, D. E. 1981, Ap. J., 243, 81 .

Collin-Souffrin, S., Dumont, S., Heidmann, N., and Joly, M. 1980, Astr. Ap., 83, 190.

Dalgarno, A., and McCray, R. 1972, Ann. Rev. Astr. Ap., 10, 375.

Daltabuit, E., MacAlpine, G. M., and Cox, D. 1978, Ap. J., 219, 372.

Dayidson, K. 1972, Ap.J., 171, 213. 1973, Ap.J., 181, 1 . 1977, Ap.J., 218, 20

Davidson, K., and Netzer, H. 1979, Rev. Mod. Phys., 51, 715.

de Vaucouleurs, G. 1979, A. J., 84, 1270.

Dopita, M. A. 1977, Ap. J. Suppl., 33, 437.

Fabian, A. C., Pringle, J., and Rees, M. 1976, M.N.R.A.S., 175, 43

Feigelson, E., Schreier, E., Delvaille, J., Giacconi, R., Grindlay, J., and Lightman, A. 1981, Ap. J., 251, 31.

Feldman, F. R., and MacAlpine, G. M. 1978, Ap. J., 221, 486.

Ferland, G. J. 1981, Ap. J., 249, 17.

Ferland, G. J., and Mushotzky, R. F. 1982, Ap. J., 262, 564.

Ferland, G. J., and Netzer, H. 1983, Ap. J., 264, 105.

Ferland, G. J., Netzer, H., and Shields, G. A. 1979, Ap. J., 232, 382

Fesen, R. A., and Kirshner, R. A. 1982, Ap. J., 258, 1.

Ginzburg, V. I., and Syrovatskii, S. I. 1964, The Origin of Cosmic Rays (Oxford: Pergamon)

Graham, J. A., and Price, R. M. 1981, Ap. J., 247, 813.

Grandi, S., and Osterbrock, D. E. 1978, Ap. J., 220,783

Guilbert, P. W., Fabian, A. C., and McCray, R. 1983, Ap. J., 266, 466.

Halpern, J. P., and Grindlay, J. E. 1980, Ap. J., 242, 1041.

Hatchett, S., Buff, J., and McCray, R. 1976, Ap. J., 206, 847

Heckman, T. M. 1980, Astr. Ap.,87, 152.

Heckman, T. M., and Balick, B. 1979, Astr. Ap., 79, 350

Heckman, T. M., Miley, G. K., Balick, B., van Breugel, W. J. M., and Butcher, H. R. 1982, Ap. J., 262, 529 .

Henry, R. B. C., and MacAlpine, G. M. 1982, Ap. J., 258, 11.

Hubbard, E. N., and Puetter, R. C. 1984, preprint.

Hummer, D. G. 1962, M.N.R.A.S., 125, 21.
Jackson, J. D. 1975, Classical Electrodynamics (New York: Wiley).

Kaler, J. B., Aller, L. H., Czyzak, S. J., and Epps, H. W. 1976, Ap. J. Suppl., 31, 163.

Kallman, T. R., and McCray, R. 1982, Ap. J. Suppl., 50, 263.

Kellermann, K. I. 1966, Ap. J., 146, 621.

Krolik, J., McKee, C., and Tarter, C. 1981, Ap. J., 249, 422

Kwan, J., and Krolik, J. 1981, Ap. J., 250, 478.

Lea, S., and Holman, G. 1978, Ap. J., 222, 29.

Longair, M. S. 1981, High Energy Astrophysics (Cambridge: Cambridge University Press).

Lotz, W. 1967, Ap. J. Suppl., 14, 207.

MacAlpine, G. M. 1972, Ap. J., 175, 11.

Mathews, W. G. 1974, Ap. J., 189, 23.

Mendoza, C. 1983, in IAU Symposium 103, Planetary Nebulae, ed. D. R Flower(Dordrecht: Reidel), p. 143.

Mihalas, D. 1978, Stellar Atmospheres (San Francisco: Freeman).

Miley, G. K. Heckman, T. M., Butcher, H. R., and van Breugel, W. J. M. 1981, Ap. J.(Letters), 247, L5.

Miley, G. K., and Miller, J. S. 1979, Ap. J. (Letters), 228, L55.

Mushotzky, R. F. 1982, Ap.J., 246, 92.

Netzer, H. $1975, M$ N R A S 171, 395. 1982, M.N.R.A.S., 198, 589

Nussbaumer, H., Pettini, M., and Storey, P. J. 1981, Astr. Ap., 102, 351.

Nussbaumer, H., and Storey, P. 1983, Astr. Ap., 126, 75.

Osterbrock, D. E. 1969, Ap. Letters, 4, 57. 1974, Astrophysics of Gaseous Nebulae (San Francisco: Freeman). 1981, Ap. J., 246, 696.

. 1983, in IAU Symposium 103, Planetary Nebulae, ed. D. R. Flower (Dordrecht: Reidel), p. 473.

1984, Quart. J. R.A.S., in press.

Pacholczyk, A. G. 1970, Radio Astrophysics (San Francisco: Freeman).

Péquignot, D. 1984, Astr. Ap., in press.

Phillips, M. M. 1979, Ap. J.Suppl., 39, 377.

Ptak, R., and Stoner, R. 1973, Ap. J.(Letters), 179, L89.

Rossi, B. 1952, High-Energy Particles (New York: Prentice-Hall)

Saslaw, W. C., Tyson, J. A., and Crane, P. 1978, Ap. J., 222, 435.

Scott, J. S., Holman, G. D., Ionson, J. A., and Papadopoulos, K. 1980, Ap. J., 239, 769 .

Shields, G. A. 1974, Ap. J., 191, 309.

1978, in Pittsburgh Conference on BL Lac Objects (Pittsburgh: University of Pittsburgh), p. 321.

Shull, J. M. 1979, Ap. J., 234, 761.

Sofia, S. 1973, Ap. J. (Letters), 179, L35.

Spitzer, L. 1962, Physics of Fully Ionized Gases (New York; Wiley).

Spitzer, L., and Tomasko, M. G. 1968, Ap. J., 152,971.

Tohline, J. E., and Osterbrock, D. E. 1982, Ap. J. (Letters), 252, L49.

Tucker, W. H., and Rosner, R. 1983, Ap. J., 267, 547.

Ulvestad, J. S., Wilson, A. S., and Sramek, R. 1981, Ap. J., 247, 419.

van Breugel, W., Heckman, T., Butcher, H., and Miley, G. 1984, Ap. J., 277, 82.

Weisheit, J., Shields, G., and Tarter, C. 1981, Ap. J., 245, 406.

Williams, R. E. $1967, A p . J ., 147,556$.

Wilson, A. 1974, M.N.R.A.S., 166, 617

1983, in Highlights in Astronomy, Vol. 6, ed. R. M. West (Dordrecht: Reidel), p. 467.

GARY J. FERLAND: Department of Physics and Astronomy, University of Kentucky, Lexington, KY 4056

RichaRd F. MuSHOTZKY: NASA/Goddard Space Flight Center, Code 661, Greenbelt, MD 20771 